\title{
Integration of Inverse Supercritical Fluid Extraction and Miniaturized 2 Asymmetrical Flow Field-Flow Fractionation for the Rapid Analysis of ${ }_{3}$ Nanoparticles in Sunscreens
}

\author{
${ }_{4}$ David Müller, ${ }^{*}, \dagger$, , I $\odot$ Margarida Nogueira, ${ }^{\dagger}$ Stefano Cattaneo, ${ }^{\dagger}$ Florian Meier, ${ }^{\S}$ Roland Drexel, ${ }^{\S}$ \\ ${ }_{5}$ Catia Contado," Antonella Pagnoni, ${ }^{\text {I Tjerk de Vries, }}{ }^{\perp}$ Dror Cohen, ${ }^{\#}$ Meital Portugal-Cohen, ${ }^{\#}$ \\ 6 and Andrew deMello \\ $7^{\dagger}$ Centre Suisse d’Electronique et de Microtechnique (CSEM), Bahnhofstrasse 1, 7302 Landquart, Switzerland \\ 8 Institute for Chemical and Bioengineering, Department for Chemistry and Applied Biosciences, ETH Zürich, Vladimir-Prelog-Weg \\ 9 1, 8093 Zürich, Switzerland \\ $10{ }^{\$}$ Postnova Analytics GmbH, Max-Planck-Strasse 14, 86899 Landsberg am Lech, Germany \\ 11 "Department of Chemical and Pharmaceutical Sciences, University of Ferrara, Via L. Borsari 46, 44121 Ferrara, Italy \\ ${ }_{12}{ }^{\perp}$ Feyecon Carbon Dioxide Technologies, Rijnkade 17a, 1382 GS Weesp, The Netherlands \\ ${ }_{13}$ "AHAVA Dead Sea Laboratories, 1 Arava Street, 70150 Lod, Israel
}

14 ABSTRACT: We report the use of inverse supercritical fluid

15 extraction (SFE) and miniaturized asymmetrical flow field-flow

16 fractionation (mAF4) for the preparation and subsequent 17 analysis of titanium dioxide nanoparticles in model and 18 commercial sunscreens. The approach allows for the fast and 19 reliable fractionation and sizing of $\mathrm{TiO}_{2}$ nanoparticles and their 20 quantitation in commercial products. This new method 21 represents a powerful and efficient tool for the verification of 22 nanoparticle content in a wide range of matrixes, as demanded 23 by recently introduced regulatory requirements. Furthermore, 24 the use of carbon dioxide as an environmentally friendly solvent 25 is in line with the increasing need for ecologically compatible 26 analytical techniques.

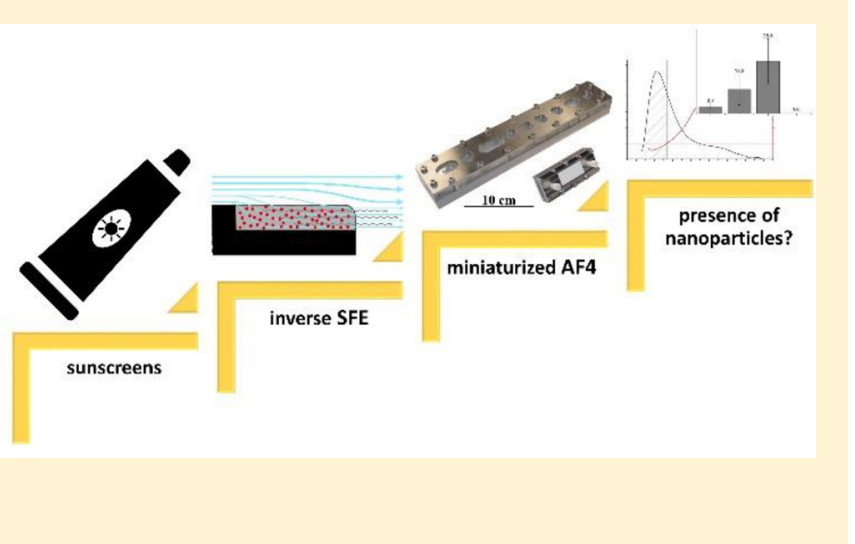

${ }_{27} \mathrm{R}$ ecently introduced European Union regulations on 28 cosmetic products require that all ingredients present in 29 the form of nanomaterials be clearly indicated as such in the list 30 of ingredients. ${ }^{1}$ This requirement calls for the development of 31 comprehensive analytical procedures to ensure manufacturer 32 compliance. The analysis of nanoparticles in complex media, 33 consisting of a complex multicomponent matrix, ${ }^{2}$ is a 34 multifaceted challenge involving multiple component processes 35 for sample pretreatment, separation of the engineered nano36 particles (ENPs) from the matrix, separation of nanoparticles 37 on the basis of their size, and chemical analysis. To this end, 38 Wagner et al. recently presented such a categorization in a 39 generic sample preparation scheme for inorganic ENPs within 40 complex matrixes. ${ }^{3}$ Such generic procedures, which also 41 integrate appropriate quality criteria to confirm the applicability 42 of the suggested methods, are urgently needed for standardized 43 and systematic development of processes for the separation and 44 analysis of ENPs in complex matrixes. In this respect, the most 45 pressing needs are the development of new analytical 46 techniques for extraction, cleanup, and separation with a view 47 to improving analytical speed, sensitivity, and specificity. ${ }^{4}$ One 48 of the most challenging components of the Wagner scheme is the reduction in complexity of the ENP containing sample, 49 either through extraction of nanoparticles from their environ- 50 ment or through simplification of the matrix by removal of 51 excipients that interfere with subsequent analysis. There are 52 several techniques that are frequently used for the isolation of 53 nanoparticles from such matrixes, including acid digestion 54 (assisted by heat, sonication, or microwaves),,$^{3,5-8}$ colloidal 55 extraction, ${ }^{3,9}$ or various treatments with organic solvents. ${ }^{7,10,11} 56$ However, all these processes are complex and time-consuming. 57 Many also completely destroy the particulate nature of the 58 samples, rendering particle size analysis impossible, or have a 59 considerable environmental impact due to the extensive use of 60 harmful solvents. ${ }^{12,13}$ Accordingly, the simplification of sample 61 preparation workflows as well as a reduction in solvent 62 requirements, are highly desirable for the analysis of nano- 63 particle-containing samples.

To this end, we present a novel method for the analysis of 65 $\mathrm{TiO}_{2}$ nanoparticles in commercial sunscreens, comprising two 66

Received: November 2, 2017

Accepted: February 7, 2018

Published: February 7, 2018 


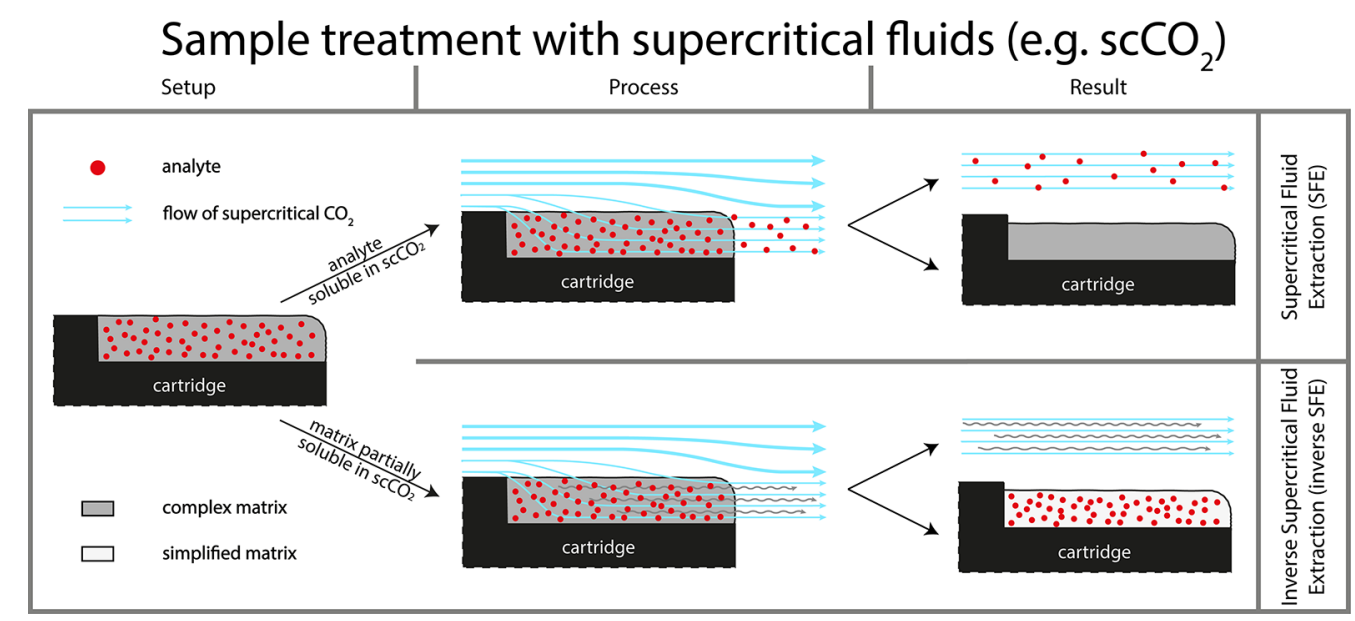

Figure 1. Principles of SFE (top) and inverse SFE (bottom). The setup used for both processes is identical, the primary difference lying in the selection of analyte and matrix and whether the substance of interest is accordingly extracted or left within the simplified matrix.

67 analytical procedures. We herein propose the use of inverse 68 supercritical fluid extraction (SFE) to simplify complex matrixes 69 containing ENPs while maintaining their particulate nature. To 70 date, the technique of inverse SFE has primarily been used for 71 the isolation of nonpolar pharmaceutical formulations from 72 polar analytes. ${ }^{13-15}$ In a recent publication, we presented proof73 of-concept experiments that suggested the utility of the 74 technique as a sample pretreatment tool for nanoparticle75 containing samples. Specifically, a single model sunscreen was 76 treated and subsequently analyzed by asymmetrical flow field77 flow fractionation (AF4) in addition to UV and MALS 78 (multiangle light scattering) detectors and the use of more 79 sophisticated transmission electron microscopy (TEM) anal80 ysis. $^{16}$ In the present work, we aim to provide a thorough 81 quantitative analysis of several model and commercial 82 sunscreens loaded with ENPs at varying concentrations. In 83 addition to the measurement of processed materials using $84 \mathrm{mAF} 4$, the ENP content was also analyzed using element85 specific tools such as inductively coupled mass spectrometry 86 (ICP-MS) and inductively coupled plasma atomic emission 87 spectroscopy (ICP-AES). Field-flow fractionation methods are 88 ideal for such measurements due to the gentle forces used to 89 induce analyte retention and prevent particle alteration. ${ }^{17}$ 90 Crucially, the miniaturized AF4 platform reduces both 91 processing times and eluent consumption. ${ }^{18}$

\section{MATERIALS AND METHODS}

93 Samples. Titanium Dioxide Nanoparticle Standards. A 94 titanium dioxide $\left(\mathrm{TiO}_{2}\right)$-nanoparticle dispersion, AERODISP 95 W 740 X (40\% w/w, EVONIK Industries, Hanau, Germany) 96 was diluted and prepared according to a protocol described 97 elsewhere $^{16}$ to yield a final particle concentration of $0.2 \mathrm{mg} /$ $98 \mathrm{~mL}$.

99 Model Sunscreen. The novel sample preparation method 100 was initially applied to three complex sunscreen models 101 containing different $\mathrm{TiO}_{2}$ nanoparticle concentrations. Creams 102 were produced individually according to protocols described 103 elsewhere. ${ }^{16}$ In the final step, $0.5,2.5$, and $12.5 \% \mathrm{w} / \mathrm{w}$ of a 104 AERODISP W $740 \mathrm{X} \mathrm{TiO}_{2}$ nanoparticle dispersion (40\% w/w, 105 EVONIK Industries, Essen, Germany), as well as the excipients 106 Dow Corning 1503 (Dow Corning Corporation, Midland, MI, 107 United States) and Euxyl PE 9010 (Schülke \& Mayr GmbH, 108 Norderstedt, Germany) were added to each cream, resulting in a $\mathrm{TiO}_{2}$ particle concentration of $0.2,1.0$, and $5.0 \% \mathrm{w} / \mathrm{w}, 109$ respectively. All creams were homogenized for $5 \mathrm{~min}$ at 4000110 $\mathrm{rpm}$ before being loaded into tubes and stored at room 111 temperature.

Commercial Sunscreens. Five different commercial creams 113 were used to assess our sample preparation method: one cream 114 with a sun protection factor (SPF) of 15 (NiveaCream15), two 115 creams of different brands with a SPF of 30 (GarnierCream30 116 and NiveaCream30), one cream with an SPF of 50117 (CoopCream50), and a sun protection spray with an SPF of 118 30 (SherpaSpray30). The first three samples contain $\mathrm{TiO}_{2} 119$ nanoparticles listed as an ingredient according to European 120 Union legislation, whereas the latter two do not list any 121 nanoparticles as ingredients.

122

Sample Treatment. Supercritical fluids are well-suited for 123 extraction processes due to their minimal surface tension, low 124 viscosities, and gas-like diffusivities, which allow for thorough 125 sample penetration while maintaining the structure of the 126 residual material. ${ }^{13}$ The most obvious choice for the current 127 application is supercritical carbon dioxide $\left(\mathrm{scCO}_{2}\right)$ because 128 almost all chemical excipients found in emulsion-based 129 cosmetic products are highly soluble in $\mathrm{CO}_{2} \cdot \mathrm{scCO}_{2}$ is 130 chemically inert $^{19}$ as well as being nontoxic and nonflam- 131 mable $\mathrm{e}^{20}$ and is commonly used in the extraction of small and/ 132 or nonpolar molecules from natural materials. ${ }^{21-24}$ In this 133 regard, common applications include the extraction of essential 134 oils from herbs and spices, ${ }^{25,26}$ the removal of caffeine from 135 coffee beans, ${ }^{27,28}$ and the extraction and analysis of 136 antioxidants, preservatives, and sunscreen agents in cosmet- 137 ics. $^{29,30}$ In all these applications, however, the analyte itself is 138 soluble in $\mathrm{CO}_{2}$, with SFE being used to dissolve and remove 139 the analyte from the matrix (upper panel of Figure 1). In this $140 \mathrm{fl}$ work, however, inverse SFE is used to simplify the matrix by 141 removal of only unwanted components (see lower part of 142 Figure 1). Put simply, the supercritical fluid permeates the 143 matrix, dissolving fatty components and leaving behind a 144 simplified matrix. Once complete, any residual $\mathrm{CO}_{2}$ is simply 145 removed by lowering the pressure below the critical threshold 146 and returning to ambient conditions. The remaining sample 147 material consists of the polar components of the matrix (e.g., 148 thickening agents) along with any nanoparticles present. 149 Residue can be easily rewetted and subsequently dispersed in 150 an aqueous matrix for subsequent analysis. 
152 Supercritical $\mathrm{CO}_{2}$ Sample Treatment. Details of the 153 analytical method have been described previously, ${ }^{16}$ and thus, 154 only the conditions and experimental setup are described 155 herein. For our measurements, the sunscreen was placed on a 156 Teflon cartridge surrounded by a stainless-steel holder. The 157 Teflon part contained a small recess, forming in a cavity with 158 dimensions of $60 \times 10 \times 0.2 \mathrm{~mm}$ and accommodating $100 \mathrm{mg}$ 159 of cream. To ensure a reproducible sample volume, excess 160 sunscreen was removed using a spatula. The Teflon cartridge 161 was then removed from its holder, weighed, and placed in a 162 custom-made extraction vessel. The setup was equipped with a 163 high-pressure $\mathrm{CO}_{2}$ pump, a pressure/flow regulating system, 164 and a vertically mounted extraction vessel. A custom-made 165 support was used to place two cartridges at once in the 166 extraction vessel. Data indicated no significant difference 167 between each position; however, only the measurements from 168 one cartridge position (closer to the $\mathrm{CO}_{2}$ inlet) are shown. The 169 sample was then subjected to a constant $\mathrm{scCO}_{2}$ flow of $80 \mathrm{~g}$ $170 \mathrm{~min}^{-1}$ for $30 \mathrm{~min}$ at $40{ }^{\circ} \mathrm{C}$ and 131 bar. After extraction, the 171 cartridge was removed and placed in a $15 \mathrm{~mL}$ tube for storage. 172 Before the measurement, $10 \mathrm{~mL}$ of a solution of $0.2 \%(\mathrm{v} / \mathrm{v})$ 173 NovaChem (Postnova Analytics GmbH, Landsberg am Lech, 174 Germany) in ultrapure water (Milli-Q Billerica, United States) 175 was added. NovaChem is a mixture of nonionic and ionic 176 detergents that helps prevent particle agglomeration. The tube 177 was vortexed and sonicated at maximum power $(132 \mathrm{kHz})$ for 17830 min using a ultrasonic bath (Ultrasonic Cleaner USC-THD/ 179 HF, VWR, Radnor, Pennsylvania, United States) previously 180 cooled to a temperature of $25{ }^{\circ} \mathrm{C}$ to further reduce eventual 181 particle agglomerates. All extractions were performed in 182 triplicate $(n=3)$.

183 Microwave Assisted Digestion. Between 0.15 and $0.2 \mathrm{~g}$ of 184 each sample was deposited in a Teflon vessel (Milestone Inc., 185 Shelton, United States), to which $6 \mathrm{~mL}$ of nitric acid (ultrapure 186 p.a. $>65 \%$, Sigma-Aldrich S.R.L., Milano, Italy), $1 \mathrm{~mL}$ of $\mathrm{H}_{2} \mathrm{O}_{2}$ 187 (30\% RPE, Carlo Erba Reagents S.r.l., Cornaredo, Italy), and 3 $188 \mathrm{~mL}$ of HF (39.5\% RPE, Carlo Erba Reagents S.r.l., Cornaredo, 189 Italy) were added. The samples were then digested in the 190 microwave oven (Ethos 900 Milestone, rotor HPR 1000/6M, 191 Milestone Inc., Shelton, United States) according to the 192 following program: $1 \mathrm{~min}$ at $250 \mathrm{~W}$ and $120^{\circ} \mathrm{C}, 1 \mathrm{~min}$ at 0 $193 \mathrm{~W}$ and $120^{\circ} \mathrm{C}, 5 \mathrm{~min}$ at $250 \mathrm{~W}$ and $140{ }^{\circ} \mathrm{C}, 4 \mathrm{~min}$ at $400 \mathrm{~W}$ 194 and $220^{\circ} \mathrm{C}, 3 \mathrm{~min}$ at $550 \mathrm{~W}$ and $220^{\circ} \mathrm{C}, 5 \mathrm{~min}$ at $300 \mathrm{~W}$ and $195220^{\circ} \mathrm{C}$. The vessel was then left to cool for at least $10 \mathrm{~min}$ 196 before it was opened, and $1.5 \mathrm{~g}$ of boric acid (99.97\% trace 197 metal basis, Sigma-Aldrich S.R.L., Milano, Italy) was added. 198 The solution was then returned into the microwave for $5 \mathrm{~min}$ at 199300 W. Finally, the vessel was cooled to ambient temperature, 200 and the contents were transferred to a volumetric flask where 201 ultrapure water was added to reach the final volume of $50 \mathrm{~mL}$. 202 All creams dissolved quickly with no visible residue; the final 203 solutions were clear and transparent. All digestions were 204 performed in triplicate $(n=3)$.

205 Element-Specific Batch Analysis and Recovery Rate. For 206 the batch-analysis of samples, two different element-specific 207 detectors were used, namely ICP-AES and ICP-MS. For the 208 evaluation of recovery rates, the detected concentrations were 209 compared to those of the samples prepared by direct 210 microwave-assisted digestion (without any treatment by inverse 211 SFE) followed by the corresponding element-specific method. 212 These procedures have previously been shown to allow 213 recovery rates of $98.2 \pm 2.2 \%$ for the $\mathrm{ICP}_{-\mathrm{AES}^{5}}$ and $101 \pm$ $2142 \%$ for the ICP-MS. ${ }^{7}$
Inductively Coupled Plasma-Atomic Emission Spectrom- 215 etry. Titanium determinations were carried out on a 216 PerkinElmer Optima 3100 XL (PerkinElmer Italia S.p.A, 217 Milano, Italy) ICP-AES equipped with an axial torch, 218 segmented array charge-coupled device detector, and Low- 219 Flow GemCone nebulizer with cyclonic spray chamber for 220 sample introduction and choosing, among the several wave- 221 lengths, the readings at $337.279 \mathrm{~nm}$. The plasma conditions 222 used were an RF power of $1350 \mathrm{~W}$ applied to the plasma and 223 flow rates of $15 \mathrm{~L} \mathrm{~min}^{-1}$ for the plasma gas and $0.5 \mathrm{~L} \mathrm{~min}^{-1}$ for 224 the auxiliary gas with a nebulizer gas flow of $0.65 \mathrm{~L} \mathrm{~min}^{-1}$. The 225

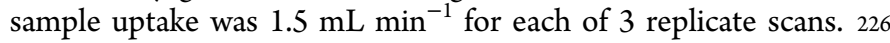
The diluted standard solutions were prepared from an 227 elemental standard solution (1000 $\mathrm{mg} \mathrm{L}^{-1} \mathrm{Ti}$, monoelement 228 standard solution, Carlo Erba, Italy). Titanium quantification 229 limits were evaluated each time from the calibration curves, and 230 the values ranged between $0.54-0.59 \mathrm{mg} \mathrm{L}^{-1}$.

Inductively Coupled Plasma-Mass Spectrometry. Tita- 232 nium contents were also determined using an Agilent 7900233 (Agilent Technologies, Santa Clara, United States) ICP-MS. 234 Sample introduction was carried out with a concentric glass 235 Micromist nebulizer, quartz glass spray chamber and a quartz 236 glass axial torch. A RF power of $1550 \mathrm{~W}$ and an argon gas flow 237 of $15 \mathrm{~L} \mathrm{~min}^{-1}$ with a $0.9 \mathrm{~L} \mathrm{~min}^{-1}$ auxiliary gas flow and a carrier 238 gas flow of $1.05 \mathrm{~L} \mathrm{~min}^{-1}$ were used. The sample uptake flow 239 rate was $1.38 \mathrm{~mL} \mathrm{~min}^{-1}$ with a stabilization time of $60 \mathrm{~s}$. The 240 titanium isotopes were recorded with an integration time of 0.5241 s. All measurements were performed in triplicate in He-mode to 242 remove polyatomic interferences by introducing a helium flow 243 of $4.3 \mathrm{~mL} \mathrm{~min}^{-1}$ to the collision cell. The calibration was 244 performed with a multielemental standard solution of $1 \mathrm{mg} \mathrm{L}^{-1} 245$ titanium (CPAchem Ltd., Stara Zagora, Bulgaria), and a 246 detection limit of $13 \pm 4 \mu \mathrm{g} \mathrm{L}^{-1}$ was obtained according to 247 DIN32645 (calibration curve method). Prior to analysis, all 248 samples were diluted with a $2 \%(\mathrm{v} / \mathrm{v}) \mathrm{HNO}_{3}$ solution. The 249 isotopes ${ }^{48} \mathrm{Ti}$ and ${ }^{49} \mathrm{Ti}$ were always measured simultaneously. 250 However, only the data from ${ }^{49} \mathrm{Ti}$ are shown.

251

Miniaturized AF4 with a Multi-Detector Array. 252 Instrumentation and Carrier Liquid. Samples treated with 253 inverse SFE were further separated and analyzed using an 254 asymmetrical flow field-flow fractionation system (AF4) from 255 Postnova Analytics GmbH (AF2000 MF, Postnova Analytics 256 $\mathrm{GmbH}(\mathrm{PN})$, Landsberg am Lech, Germany), incorporating an 257 autosampler (PN5300), channel thermostat (PN4020), UV 258 (PN3211) and multi-angle light scattering MALS (PN3621, 21259 angles) detectors. A miniaturized AF4 cartridge with a tip to tip 260 length of $7 \mathrm{~cm}$ (S-AF4-CHA-631, a similar design is further 261 described in ref 18) and incorporating a small $10 \mathrm{kDa} 262$ regenerated cellulose membrane $(20 \times 80 \mathrm{~mm}, \mathrm{Z}-263$ AF4_MEM-635-10KD) was used for samples of the model 264 sunscreens and a miniaturized $10 \mathrm{kDa}$ polyether sulfone (PES) 265 membrane $(20 \times 80 \mathrm{~mm}, \mathrm{Z}-\mathrm{AF} 4$ _MEM-631-10KD) for the 266 commercial sunscreens. Furthermore, a $350 \mu$ m-thick Mylar 267 spacer was used for all measurements. UV detection was 268 performed at $254 \mathrm{~nm}$, and the measured UV signal was used to 269 correlate concentration with particle size. The MALS detector 270 provided the gyration radius of the particles exiting the 271 miniaturized AF4 (mAF4) separation cartridge (calculated 272 using the random coil model). All presented UV data were 273 collected with the UV detector alone, and all radii of gyration 274 data were determined from the angular dependent light 275 scattering signals obtained via MALS detection. The eluent 276 was prepared using filtrated ultrapure water, to which $0.2 \%$ (v/ 277 
Table 1. Titanium Dioxide Concentrations Measured after Different Sample Treatments and Subsequent Element-Specific Analysis with Model Sunscreens

\begin{tabular}{|c|c|c|c|c|c|c|c|c|}
\hline & \multicolumn{4}{|c|}{ sample preparation: microwave digestion } & \multicolumn{4}{|c|}{ sample preparation: inverse SFE } \\
\hline & \multicolumn{2}{|c|}{ analytical method: ICP-MS } & \multicolumn{2}{|c|}{ analytical method: ICP-AES } & \multicolumn{2}{|c|}{ analytical method: ICP-MS } & \multicolumn{2}{|c|}{ analytical method: ICP-AES } \\
\hline & conc $(\%)$ & SD & conc $(\%)$ & SD & conc $(\%)$ & SD & conc $(\%)$ & SD \\
\hline model sunscreen, $0.2 \% \mathrm{TiO}_{2}$ & 0.19 & 0.02 & 0.21 & 0.01 & 0.25 & 0.03 & 0.21 & 0.03 \\
\hline model sunscreen, $1.0 \% \mathrm{TiO}_{2}$ & 1.02 & 0.01 & 0.99 & 0.01 & 1.16 & 0.05 & 1.05 & 0.03 \\
\hline model sunscreen, $5.0 \% \mathrm{TiO}_{2}$ & 5.17 & 0.06 & 4.95 & 0.07 & 5.21 & 0.06 & 5.3 & 0.3 \\
\hline
\end{tabular}

$278 \mathrm{v}$ ) filtered NovaChem was added. The injection volume was set 279 to $5 \mu \mathrm{L}$ for the highly concentrated standard $\mathrm{TiO}_{2}$ nano280 particles, $10 \mu \mathrm{L}$ for all model sunscreens and the 281 GarnierCream30 (highest $\mathrm{TiO}_{2}$ content of the commercial 282 samples), and to $20 \mu \mathrm{L}$ for all other commercial creams. Prior 283 to analysis, the samples from the model sunscreens were diluted $2841: 4$ in a solution of $0.2 \%(\mathrm{v} / \mathrm{v})$ NovaChem in ultrapure water 285 and then again sonicated at maximum power $(132 \mathrm{kHz})$ for 15 $286 \mathrm{~min}$. Dilution is necessary to prevent overloading effects, which 287 may cause peak shifts and lead to particle agglomeration. The 288 commercial creams were transferred to a glass vial and 289 sonicated for $30 \mathrm{~min}$ without further dilution. The temperature 290 of both the autosampler and the channel thermostat were set to $29125{ }^{\circ} \mathrm{C}$. Separations and analysis were performed in quad292 ruplicate $(m=4)$ for each extraction (each cream was sampled 293 in triplicate $n=3$ ) and three out of four measurements per 294 extraction were selected for further investigations. To 295 compensate the baseline drift, UV data was corrected by 296 subtracting a blank run signal measured after injection of pure 297 eluent. For the commercial sunscreens, the baseline corrected 298 UV signal was also divided through the original cream weight, 299 eliminating the influence of different sample amounts. The data 300 acquisition and MALS calculations were performed using the 301 AF2000 Control Unit software (Postnova Analytics GmbH, 302 Landsberg am Lech, Germany), and further evaluations (curve 303 subtractions, etc.) were performed using OriginPro 9.1 304 (OriginLab Corporation, United States).

305 Elution Profile. An optimized focusing and elution method 306 was developed to ensure reproducible analysis. The focusing 307 step of the selected elution profile was commenced with a 4 308 min-long injection flow of $0.15 \mathrm{~mL} \mathrm{~min}^{-1}$ and a cross-flow of $3090.30 \mathrm{~mL} \mathrm{~min}^{-1}$. After a $30 \mathrm{~s}$-long transition time, elution started 310 with a constant cross-flow of $0.30 \mathrm{~mL} \mathrm{~min}^{-1}$ for an additional $31130 \mathrm{~s}$, followed by an exponentially decreasing cross-flow 312 (exponent: 0.2 ), reaching a final value of $0 \mathrm{~mL} \mathrm{~min}^{-1}$ after $31330 \mathrm{~min}$. The run was completed with a $10 \mathrm{~min}$ long rinsing step 314 to check for additional particle release. To ensure a stable 315 signal, the detector flow rate was maintained at $0.35 \mathrm{~mL} \mathrm{~min}^{-1}$, 316 with the other flows adjusted accordingly.

317 Determination of Relative Particle Amounts. For the 318 commercial sunscreens, the relative number of particles with a 319 gyration diameter of less than a $100 \mathrm{~nm}$ was estimated by 320 integrating the area under the curve of the UV trace between 321 the end of the void peak $(6.25 \mathrm{~min})$ and the elution time 322 corresponding to a gyration diameter of $100 \mathrm{~nm}$, as calculated 323 from the MALS measurements.

\section{RESULTS AND DISCUSSIONS}

325 Method Evaluation with Model Sunscreen. Recovery 326 Rate. One of the most relevant figures of merit when separating 327 ENPs from complex matrixes is the recovery rate. ${ }^{3}$ This was 328 investigated with ICP-AES and ICP-MS for three model 329 sunscreens that were independently treated with both a standard procedure based on microwave-assisted digestion 330 and inverse SFE.

As shown in Table 1, slightly higher concentrations of $\mathrm{TiO}_{2} 332 \mathrm{tl}$ were obtained for samples processed with $\mathrm{scCO}_{2}$, resulting in 333 recovery rates of $104 \pm 4 \%$ for the ICP-AES and $115 \pm 17 \%$ for 334 the ICP-MS. This apparent increase in concentration is most 335 likely due to solvent evaporation during sample preparation for 336 the inverse SFE treatment: the amount of cream to be 337 processed by $\mathrm{ScCO}_{2}$ is weighed after deposition on the Teflon 338 cartridge, where it is spread into a homogeneous $200 \mu \mathrm{m}$ thin 339 layer to allow thorough sample penetration. In the time 340 between sample deposition and cartridge weighing, the sample 341 is prone to evaporation due the small amount of cream (about 342 $100 \mathrm{mg})$ and the large exposed surface $\left(600 \mathrm{~mm}^{2}\right)$. This, in 343 combination with the fact that sunscreens are often tailored for 344 fast solvent evaporation (to promote quick absorption by the 345 skin), may lead to mass losses of up to $10 \%$, shifting the 346 nanoparticle $\mathrm{w} / \mathrm{w}$ concentration toward higher values. This 347 does not occur when performing acid digestion, thereby 348 explaining the small difference in measured concentrations. 349

Particle Size Evaluation. Another important factor with 350 regard to the applicability of a sample preparation method is its 351 ability to preserve the size distribution of the nanoparticles and 352 prevent the creation of large agglomerates. To assess this, we 353 used a miniaturized AF4 cartridge to perform rapid size 354 separation and subsequent characterization by different 355 detection techniques. ${ }^{18}$ With the miniaturized cartridge and 356 the correspondingly low flow rates, solvent consumption could 357 be reduced to less than $25 \mathrm{~mL}$ per run, compared to the 100358 $\mathrm{mL}$ normally used within the standard analytical channel. The 359 complete run time with the miniaturized channel was $45 \mathrm{~min}, 360$ significantly shorter than what is needed when using standard 361 analytical cartridges, especially when taking into consideration 362 flow presetting and flushing. Besides the three model 363 sunscreens, we also measured the pure nanoparticle dispersion 364 used in their fabrication, allowing direct comparison of the size 365 of the original nanoparticles to those remaining in the 366 simplified matrix after treatment with $\mathrm{scCO}_{2}$. These data are 367 shown in Figure 2, where the solid lines depict the UV $368 \mathrm{f} 2$ fractograms for the different model sunscreens and the black 369 dashed line shows the comparative UV data for the original 370 nanoparticle suspension. The red dashed line shows the 371 averaged MALS curve for the pure dispersion, which is in 372 good agreement with the corresponding curves for the model 373 sunscreens. Peak positions in the different fractograms are in 374 close correspondence with only a small shift of the main peak 375 for the treated particles toward larger sizes. These slight shifts 376 are almost certainly due to aggregation. Nevertheless, because 377 larger particles scatter light strongly, even small amounts of 378 aggregates would result in higher signals. Accordingly, the 379 elugrams indicate the presence of only minor aggregates. The 380 very small peak occurring between 15 and 20 min most likely 381 originates from other cream excipients being slightly active in 382 


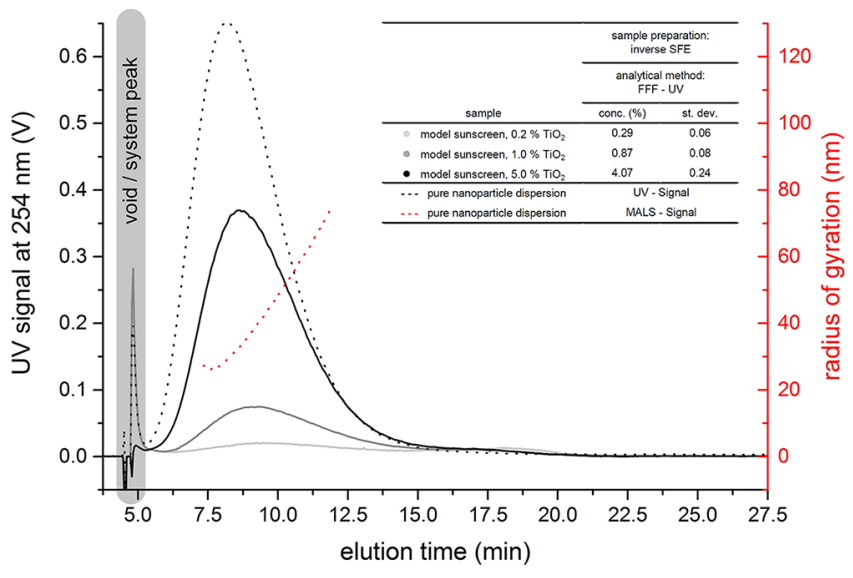

Figure 2. Fractograms of the three $\mathrm{scCO}_{2}$ treated model sunscreens. In addition, the UV signal and calculated radius of gyration from the MALS detector of the pure nanoparticle dispersion are shown, confirming the excellent correspondence between the size distributions from the original particles and those extracted from the complex sunscreen matrix.

383 the selected UV absorption range, as previously noted ${ }^{16}$ and 384 through additional ICP-MS measurements (data not shown). 385 Overall, however, the data clearly indicate excellent preserva386 tion of the size distribution of the particles between the pure 387 and treated samples.

388 Method Evaluation with Commercial Sunscreens. 389 Recovery Rate. After the sample preparation efficiency of 390 inverse SFE was thoroughly tested on model sunscreens, the 391 method was applied to commercial sunscreens. For these tests, 392 we purchased a set of five sunscreens and processed them using 393 the same protocol that was used for the three model sunscreen 394 formulations, in addition to microwave-assisted digestion and 395 the treatment with supercritical $\mathrm{CO}_{2}$ (Table 2). Significantly, 396 the $\mathrm{TiO}_{2}$ concentrations, determined after microwave digestion 397 with the two methods, are in good agreement, allowing an 398 accurate estimation of the $\mathrm{TiO}_{2}$ content in the commercial 399 formulations. The values for the CoopCream50 and the 400 SherpaSpray30 (the two creams sold as being $\mathrm{TiO}_{2}$-free) gave 401 signals consistently below the detection limit of the respective 402 analytical technique.

403 An interesting result was observed when using elemental 404 techniques to determine the $\mathrm{TiO}_{2}$ concentration after inverse 405 SFE. The value obtained with ICP-AES was approximately $79 \%$ 406 of the initial concentration, with little variation among different 407 screens. Conversely, ICP-MS data estimated 52, 37, and 44\% of 408 the corresponding initial values. The reduced recovery rate for 409 the commercial sunscreens may indicate a severe loss of $\mathrm{TiO}_{2}$ 410 during sample preparation with inverse SFE. However, it has been shown previously that organic acids can form in the 411 presence of water and $\mathrm{scCO}_{2}$ and that such acids can diminish 412 the suspension stability of $\mathrm{TiO}_{2}$ nanoparticles. ${ }^{31,32}$ The 413 treatment with inverse SFE could therefore lead to a 414 modification in the nanoparticle's surfactant properties, which 415 would result in particle agglomeration. These larger aggregates 416 are unlikely to be completely ionized by the ICP ion source, 417 thereby resulting in lower recoveries at the detector. 418 Furthermore, the different measurement process parameters 419 of ICP-MS and ICP-AES such as speed and sample uptake 420 could also yield more rapid precipitation for highly aggregated 421 particles in the case of ICP-MS, leading to lower recovery rates 422 compared to those of ICP-AES. ${ }^{33}$

423

To further investigate this issue, we performed additional 424 measurements where sunscreens processed using inverse SFE 425 were further treated with microwave-assisted digestion prior to 426 analysis with element-specific tools. This additional mineraliza- 427 tion step should allow the proper ionization of all the $\mathrm{TiO}_{2}$ left 428 after treatment with supercritical $\mathrm{CO}_{2}$, clarifying whether 429 particles were lost during inverse SFE treatment or not. $\quad 430$

The data in Table 3 report the recovery rates for the three $431 \mathrm{t} 3$ nanoparticle-containing commercial samples after employing 432

Table 3. Recovery Rates of the Three Commercial Products Containing $\mathrm{TiO}_{2}$ Nanoparticles ${ }^{a}$

\begin{tabular}{|c|c|c|c|c|}
\hline & \multicolumn{2}{|c|}{ sample preparation: inverse SFE } & \multicolumn{2}{|c|}{$\begin{array}{l}\text { sample preparation: iSFE + } \\
\text { microwave digestion }\end{array}$} \\
\hline & $\begin{array}{l}\text { analytical } \\
\text { method: } \\
\text { ICP-MS (\%) }\end{array}$ & $\begin{array}{c}\text { analytical } \\
\text { method: } \\
\text { ICP-AES (\%) }\end{array}$ & $\begin{array}{l}\text { analytical } \\
\text { method: } \\
\text { ICP-MS (\%) }\end{array}$ & $\begin{array}{l}\text { analytical } \\
\text { method: } \\
\text { ICP-AES (\%) }\end{array}$ \\
\hline NC15 & $52 \pm 16$ & $79 \pm 4$ & $106 \pm 14$ & $95 \pm 6$ \\
\hline NC30 & $37 \pm 20$ & $78 \pm 28$ & $110 \pm 8$ & $90 \pm 8$ \\
\hline GC30 & $44 \pm 7$ & $80 \pm 15$ & $68 \pm 10$ & $78 \pm 12$ \\
\hline
\end{tabular}

${ }^{a}$ Abbreviations: NC15, NiveaCream15; NC30, NiveaCream30; GC30, GarnierCream30.

only inverse SFE and after processing creams with inverse SFE 433 followed by microwave-assisted digestion. As can be seen, the 434 obtained recovery values are higher in the latter case, indicating 435 the issue is not a result of particle loss during inverse SFE but 436 rather due to incomplete ionization of the gently treated 437 samples.

Particle Size Evaluation. The aforementioned drop in 439 recovery rate may pose an issue for quantitative measurements. 440 However, one significant advantage of inverse SFE over 441 microwave-assisted digestion is its capability to preserve 442 nanoparticles during pretreatment, thus allowing further 443 investigation. In the case of microwave-assisted digestion, the 444 metals are fully solubilized, thereby destroying their particulate 445 nature and preventing further investigation. On the contrary, $446 \mathrm{f3}$

Table 2. Titanium Dioxide Concentrations Measured after Different Sample Treatments and Subsequent Element-Specific Analysis for Five Commercial Sunscreens

\begin{tabular}{|c|c|c|c|c|c|c|c|c|}
\hline & \multicolumn{4}{|c|}{ sample preparation: microwave digestion } & \multicolumn{4}{|c|}{ sample preparation: inverse SFE } \\
\hline & \multicolumn{2}{|c|}{ analytical method: ICP-MS } & \multicolumn{2}{|c|}{ analytical method: ICP-AES } & \multicolumn{2}{|c|}{ analytical method: ICP-MS } & \multicolumn{2}{|c|}{ analytical method: ICP-AES } \\
\hline & conc $(\%)$ & SD & conc $(\%)$ & SD & conc $(\%)$ & SD & conc $(\%)$ & $\mathrm{SD}$ \\
\hline NiveaCream 15 & 0.99 & 0.03 & 0.92 & 0.01 & 0.5 & 0.2 & 0.72 & 0.03 \\
\hline NiveaCream 30 & 1.9 & 0.2 & 1.76 & 0.08 & 0.7 & 0.4 & 1.4 & 0.5 \\
\hline GarnierCream 30 & 3.4 & 0.2 & 3.0 & 0.2 & 1.5 & 0.2 & 2.4 & 0.4 \\
\hline CoopCream50 & $<\mathrm{LoD}$ & & $<\mathrm{LoD}$ & & $<\mathrm{LoD}$ & & $<\mathrm{LoD}$ & \\
\hline SherpaSpray30 & $<\mathrm{LoD}$ & & $<\mathrm{LoD}$ & & $<\mathrm{LoD}$ & & $<\mathrm{LoD}$ & \\
\hline
\end{tabular}



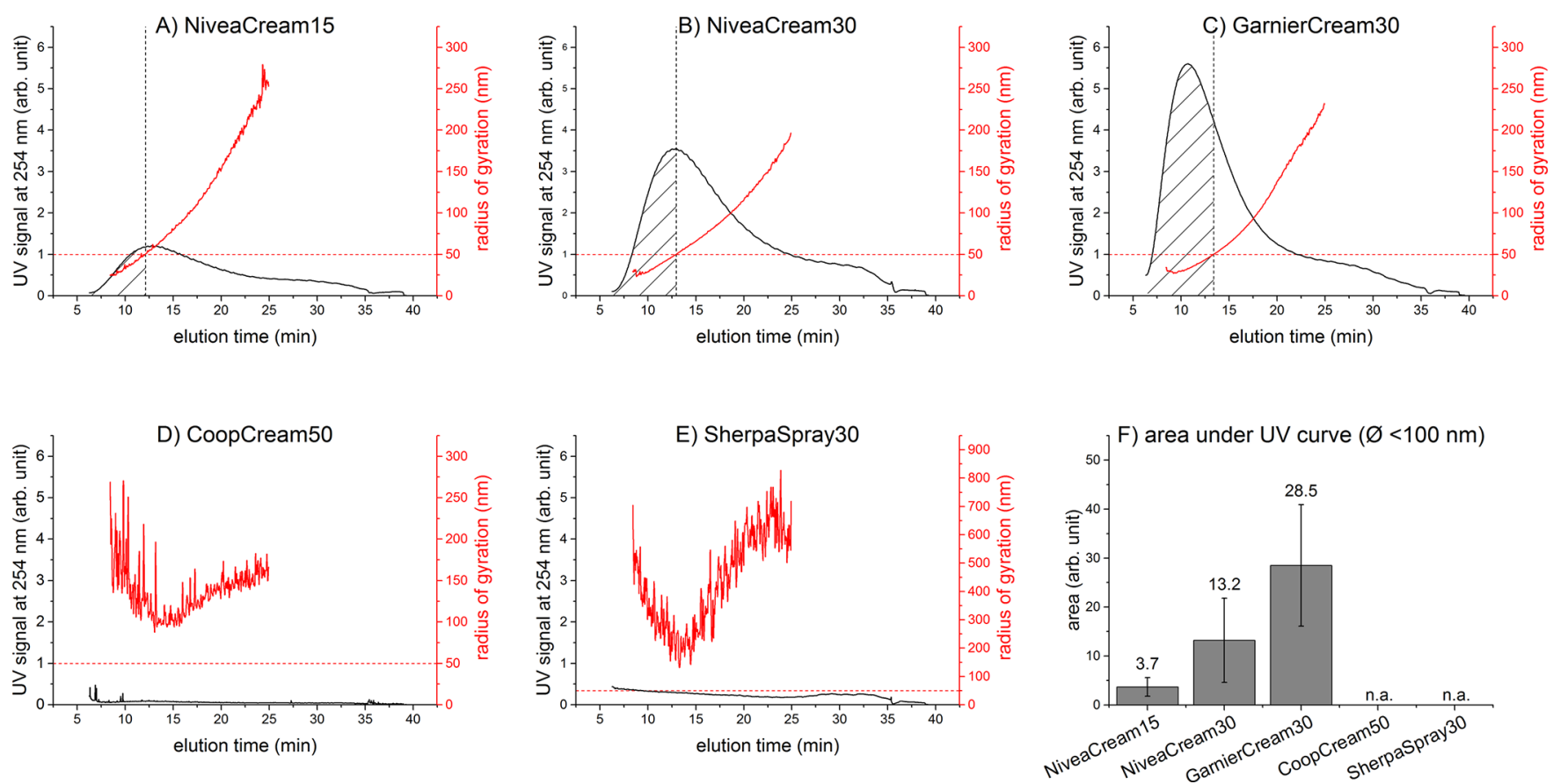

Figure 3. (A-E) Fractograms reporting the UV signal (black) and the calculated radius of gyration (red) for five commercial creams. (A-C) The first three creams clearly contain nanoparticles over a wide size range, whereas the latter two creams (D and E) show limited UV absorption and no detectable particles in the nanoscale range (and an incoherent sizing curve due to low scattering signal). Subset $\mathrm{F}$ shows the relative area under the UV curve before the threshold of an averaged gyration radius of $50 \mathrm{~nm}$ (dashed area in A-C). Error bars depict standard deviations $(n=9)$.

447 Figures 3A-E show the fractograms of resuspensions from the 448 commercial samples treated with supercritical $\mathrm{CO}_{2}$. In the case 449 of subset A-C, both UV and MALS data show a continuous 450 signal, in line with actual increase in concentration or size and 451 similar to that observed with the model samples. These three 452 creams list, as previously noted, $\mathrm{TiO}_{2}$ nanoparticles as one of 453 their ingredients. The creams not listing $\mathrm{TiO}_{2}$ or any kind of 454 nanoparticles show minimal UV absorption. In those cases, the 455 MALS detector is unable to determine a cohesive sizing curve, 456 as the low scattering signal is insufficient to allow fitting to the 457 model (Figures 3D and E). The small UV signal could be due 458 to excipients other than $\mathrm{TiO}_{2}$ nanoparticles, which may not 459 have been fully removed by inverse SFE and which are also 460 active in the selected UV absorption range.

461 Figure $3 \mathrm{~F}$ shows the area under the UV curve for all 462 commercial samples up to a determined diameter of gyration of $463100 \mathrm{~nm}$. As the MALS curves for the two later creams, 464 CoopCream50 and SherpaSpray30, never exceed this threshold, 465 no values could be calculated for those samples. Due to the 466 unknown UV absorbance of other cream components and the 467 relatively large deviations between different runs on the same 468 sample (see error bars in Figure 3F), these data cannot be used 469 for an accurate determination of $\mathrm{TiO}_{2}$ content. Nevertheless, 470 the trends are consistent with the results obtained from the 471 element-specific detection techniques, indicating that the 472 method is eminently suitable for a preliminary screening of $473 \mathrm{TiO}_{2}$ particle content.

474 Inconsistencies in the recovery rate of ICP-MS compared to 475 ICP-AES, as seen here, are generally not uncommon and have 476 been shown to become more severe if different sample 477 pretreatment are employed. ${ }^{33}$ In addition to the variations 478 between the different creams, we observed significant 479 deviations between different aliquots of the same sample. 480 This is also indicated by the standard deviations and percentage ranges shown in Tables 2 and 3 as well as the error bars of 481 Figure $3 \mathrm{~F}$, which demonstrate the challenging nature of the 482 analysis of the commercial creams compared to model 483 sunscreens. Results could be improved by extending sample 484 preparation by post-treatment stabilization of the sample, as 485 suggested by Wagner. ${ }^{3}$ These additional procedures would 486 stabilize the particles and therefore the analytical outcome as a 487 whole as well as optimize method performance for a specific 488 combination and purpose such as iSFE-mAF4-UV-MALS for 489 the determination of $\mathrm{TiO}_{2}$ particle size distributions or 490 microwave-assisted digestion of the native creams followed by 491 ICP-AES or ICP-MS for the determination of the overall $\mathrm{TiO}_{2} 492$ content.

\section{CONCLUSIONS}

494

In this study, we present a novel approach for the analysis of 495 $\mathrm{TiO}_{2}$ nanoparticles in commercial sunscreens that comprises 496 two analytical procedures. In a first step, inverse SFE was used 497 to gently remove sunscreen matrix components, resulting in 498 dried sunscreen strips containing residual $\mathrm{TiO}_{2}$ nanoparticles, 499 which were easily redispersible. With recovery rates ranging 500 between 68 and 110\%, ICP-MS and ICP-AES measurements of 501 the commercial sunscreen samples before and after treatment 502 confirmed no significant loss of $\mathrm{TiO}_{2}$ nanoparticles during the 503 sample preparation process.

504

In a second step, after redispersion in an aqueous solvent, the 505 $\mathrm{TiO}_{2}$ nanoparticles were characterized using mAF4 hyphenated 506 with UV and MALS detection. This approach allowed for fast 507 and reliable fractionation and sizing of the $\mathrm{TiO}_{2}$ nanoparticles 508 and thus an unambiguous determination of the presence or 509 absence of $\mathrm{TiO}_{2}$ particles in commercial sunscreens. The 510 obtained results were in agreement with the data from the 511 element-specific tools and with the label indications for all 512 investigated creams. 
514 The combination of inverse SFE with miniaturized AF4 515 proved to be a highly powerful and efficient tool for the 516 verification of the nanocontent of commercial sunscreens. Due 517 to its wide applicability, this analytical approach is not solely 518 limited to sunscreens but could also be used with cosmetic 519 formulations containing other inorganic particles such as zinc 520 oxide, ceria, or silver. Furthermore, the method is fully in line 521 with the increasingly compelling demand for environmentally 522 friendly analytical methods due to the use of a mild solvent $523\left(\mathrm{scCO}_{2}\right)$ in combination with reduced consumption of eluent. 524 In addition, as a pure sample preparation method, inverse SFE 525 could potentially be applied to other analytical techniques for 526 the investigation of nanoparticles in complex cosmetic matrixes, 527 including electron microscopy, nanoparticle tracking analysis or 528 single particle ICP-MS. This renders inverse SFE a universal 529 tool for the preparation of cosmetic samples, significantly 530 contributing to the quest for routine analytical methods for the 531 verification of European Union nanoparticle labeling require532 ments. ${ }^{1}$

\section{AUTHOR INFORMATION}

\section{Corresponding Author}

535 *E-mail: science@davidmueller.ch.

536 ORCID 10

537 David Müller: 0000-0003-1914-3768

538 Andrew deMello: 0000-0003-1943-1356

539 Present Address

${ }_{40}^{\text {II }}$ D.M.: Mythenstrasse 2, 6003 Luzern, Switzerland.

541 Notes

542 The authors declare no competing financial interest.

\section{ACKNOWLEDGMENTS}

544 This work was supported by the European Commission 545 Seventh Framework Programme (Project SMART-NANO, 546 NMP4-SE-2012-280779).

\section{ABBREVIATIONS}

$548 \mathrm{CO}_{2} \quad$ carbon-dioxide

$549 \mathrm{scCO}_{2}$ supercritical $\mathrm{CO}_{2}$

$550 \mathrm{AF} 4$ asymmetrical flow field-flow fractionation

551 mAF4 miniaturized AF4

552 SFE supercritical fluid extraction

553 iSFE inverse SFE

554 MALS multiangle light scattering

555 ICP-AES inductively coupled plasma atomic emission spectrometer

556 ICP-MS inductively coupled plasma mass spectrometer

\section{REFERENCES}

559 (1) EC. Report No. 342; Off. J. Eur. Union: 2009, pp 59-209.

560 (2) Calzolai, L.; Gilliland, D.; Rossi, F. Food Addit. Contam., Part A 5612012,29 (8), 1183-1193.

562 (3) Wagner, S.; Legros, S.; Loeschner, K.; Liu, J.; Navratilova, J.; 563 Grombe, R.; Linsinger, T. P. J.; Larsen, E. H.; von der Kammer, F.; 564 Hofmann, T. J. Anal. At. Spectrom. 2015, 30, 1-11.

565 (4) von der Kammer, F.; Ferguson, P. L.; Holden, P. A.; Masion, A.; 566 Rogers, K. R.; Klaine, S. J.; Koelmans, A. A.; Horne, N.; Unrine, J. M. 567 Environ. Toxicol. Chem. 2012, 31 (1), 32-49.

568 (5) Contado, C.; Pagnoni, A. Anal. Methods 2010, 2 (8), 1112-1124.

569 (6) Dan, Y.; Shi, H.; Stephan, C.; Liang, X. Microchem. J. 2015, 122, 570 119-126.

571 (7) Nischwitz, V.; Goenaga-Infante, H. J. Anal. At. Spectrom. 2012, 27 $572(7), 1084$.
(8) Tadjiki, S.; Assemi, S.; Deering, C. E.; Veranth, J. M.; Miller, J. D. 573 J. Nanopart. Res. 2009, 11 (4), 981-988.

(9) Plathe, K. L.; Von Der Kammer, F.; Hassellöv, M.; Moore, J.; 575 Murayama, M.; Hofmann, T.; Hochella, M. F. Environ. Chem. 2010, 7576 (1), 82-93.

(10) Dunford, R.; Salinaro, A.; Cai, L.; Serpone, N.; Horikoshi, S.; 578 Hidaka, H.; Knowland, J. FEBS Lett. 1997, 418 (1-2), 87-90. 579

(11) Lewicka, Z. a.; Benedetto, A. F.; Benoit, D. N.; Yu, W. W.; 580 Fortner, J. D.; Colvin, V. L. J. Nanopart. Res. 2011, 13 (9), 3607-3617. 581

(12) Capello, C.; Fischer, U.; Hungerbuhler, K. Green Chem. 2007, 9, 582 927-934. 583

(13) Moore, W. N.; Taylor, L. T. J. Pharm. Biomed. Anal. 1994, 12584 (10), 1227-1232.

(14) Messer, D. C.; Taylor, L. T. Anal. Chem. 1994, 66 (9), 1591- 586 1592.

(15) Almodovar, R. A.; Rodriguez, R. A.; Rosario, O. J. Pharm. 588 Biomed. Anal. 1998, 17, 89-93.

(16) Müller, D.; Cattaneo, S.; Meier, F.; Welz, R.; de Vries, T.; 590 Portugal-Cohen, M.; Antonio, D. C.; Cascio, C.; Calzolai, L.; Gilliland, 591 D.; de Mello, A. J. Chromatogr. A 2016, 1440, 31-36.

(17) M-M, P.; Siripinyanond, A. J. Anal. At. Spectrom. 2014, 29 (10), 593 $1739-1752$.

(18) Müller, D.; Cattaneo, S.; Meier, F.; Welz, R.; DeMello, A. Front. 595 Chem. 2015, 3 (July), 45.

(19) Scalia, S.; Simeoni, S. Chromatographia 2001, 53, 490-494 597

(20) Kaupp, G. Angew. Chem., Int. Ed. Engl. 1994, 33 (14), 1452- 598 1455.

(21) Ndiomu, D. P.; Simpson, C. F. Anal. Chim. Acta 1988, 213, 600 237-243.

(22) Hawthorne, S. B. Anal. Chem. 1990, 62 (11), 633-642

(23) Engelhardt, H.; Zapp, J.; Kolla, P. Chromatographia 1991, 32603 (11/12), 527-537.

(24) Reverchon, E.; De Marco, I. J. Supercrit. Fluids 2006, 38 (2), 605 $146-166$

(25) Goto, M.j Sato, M, Hirose, T. J Chem. Eng. Jpn. 1993, 26, 401- 607 407.

(26) Roy, B. C.; Goto, M.; Hirose, T. Ind. Eng. Chem. Res. 1996, 35609 (2), 607-612.

(27) Peker, H.; Srinivasan, M.; Smith, J.; McCoy, B. AIChE J. 1992, 611 38 (5), 761-770.

(28) Tello, J.; Viguera, M.; Calvo, L. J. Supercrit. Fluids 2011, 59613 (2011), 53-60.

(29) Lee, M. R.; Lin, C. Y.; Li, Z. G.; Tsai, T. F. J. Chromatogr. A 615 2006, 1120 (1-2), 244-251.

(30) Scalia, S. J. Chromatogr. A 2000, 870 (1-2), 199-205.

(31) Pettibone, J. M.; Cwiertny, D. M.; Scherer, M.; Grassian, V. H. 618 Langmuir 2008, 24 (13), 6659-6667.

(32) Choi, Y. S.; Nešić, S. Int. J. Greenhouse Gas Control 2011, 5 (4), 620 788-797.

(33) Iwashita, A.; Nakajima, T.; Takanashi, H.; Ohki, A.; Fujita, Y; 62 Yamashita, T. Fuel 2006, 85 (2), 257-263.

4

76

\section{.}

